\title{
Effects of Short Duration Stretching on Disuse Muscle Atrophy in Immobilized Rat Soleus Muscles
}

\author{
Minoru OKITA ${ }^{1}$, Toshiro Yoshimura ${ }^{1}$, Jiro NAKANO ${ }^{2}$, Aya SAEKI ${ }^{3}$, Ai UeHARA ${ }^{4}$, \\ Asako MinESHITA ${ }^{5}$ and Katsumi EGUCHI ${ }^{6}$ \\ ${ }^{1}$ School of Allied Medical Sciences, Nagasaki University, Nagasaki 852-8520, Japan \\ ${ }^{2}$ Department of Health Science, Graduate School of Medical Science, Hiroshima University, Hiroshima 734-8551, Japan \\ ${ }^{3}$ Nagasaki Yurino Hospital, Nagasaki 851-2103, Japan \\ ${ }^{4}$ Seiai Rehabilitation Hospital, Fukuoka 816-0955, Japan \\ ${ }^{5}$ Komonji Hospital, Fukuoka 802-0026, Japan \\ ${ }^{6}$ The First Department of Internal Medicine, Nagasaki University School of Medicine, Nagasaki 852-8501, Japan
}

\begin{abstract}
The purpose of this study was to determine whether short duration stretching is ameliorating for disuse muscle atrophy in immobilized rat soleus muscles. Eighteen male Wistar rats (age, 8 weeks; weight, $311.0 \pm 35.6 \mathrm{~g})$ were divided randomly into control $(\mathrm{n}=3)$ and experimental $(\mathrm{n}=15)$ groups. Bilateral ankles of each rat in the experimental group were fixed in full planter flexion with a plaster cast. After the experimental groups rats were immobilized for $\mathbf{4}$ weeks, animals were divided into three groups: immobilization alone (group $I, n=3$ ), stretch training for $30 \mathrm{~min} /$ day for 1 or 3 weeks after remobilization (group $S, n=6$ ), and spontaneous recovery (non stretch training) for 1 or 3 weeks after remobilization (group NS, $n=6$ ). At the end of the experimental periods, the soleus muscle was extracted from hindlimb, and the frozen sections were stained with myofibrillar adenosine triphosphatase. After 1 week of remobilization, the means of the muscle fiber diameters for type $I$ fibers in group S had increased significantly compared with group NS, but those for type II fibers in group S did not significantly differ from that for group NS. After 3 weeks of remobilization, the means of the muscle fiber diameters for types I and II fibers in group S had increased significantly compared with group NS. No difference in the fiber type distribution were observed between the experimental group. Our findings suggest that short duration stretching induces recovery from disuse muscle atrophy after joint fixation.
\end{abstract}

Key words: immobilization, disuse muscle atrophy, stretching

(J Jpn Phys Ther Assoc 4: 1-5, 2001)

Immobilization of muscles at a shortened position results in the progression of disuse atrophy, whereas immobilization in the lengthened position induces muscle growth and muscle fiber hypertrophy ${ }^{1-3)}$. When one muscle is immobilized in the lengthened position, its antagonists are placed in a shortened position resulting in deleterious changes in these muscles. Intermittent and/or short duration, rather than continuous, stretching therefore is likely to be more beneficial for preventing atrophy in patients $^{4)}$. Yamazaki et al. reported that progression of

Received: August 9, 1999

Accepted: April 1, 2000

Correspondence to: Minoru Okita, School of Allied Medical Sciences, Nagasaki University, 1-7-1 Sakamoto, Nagasaki 852-8520, Japan disuse atrophy in growing and mature rat soleus muscles can be attenuated, but not completely prevented, by maintaining the stretch position for $20 \mathrm{~min}$ a day ${ }^{5) 6}$. Williams reported that in mouse soleus muscle a $30 \mathrm{~min}$ daily stretch produced only $9 \%$ loss of muscle weight compared with a $48 \%$ loss for continuous immobilization in the shortened position for 2 weeks and that stretch prevents muscle wasting which normally occurs during plaster cast immobilization $^{7}$.

Clinically, in cases of bed rest over a long period, immobilization of a joint by a plaster cast or orthosis induces disuse muscle atrophy, and joint contracture often occurs. It is doubtful whether the competent muscle is stretched enough when its range of movement is decreased 
by joint contracture. Furthermore, there are few clinical or experimental reports on the effects of stretching on disuse muscle atrophy followed by joint contracture. The purpose of this study was to determine whether short duration stretching is ameliorating for disuse muscle atrophy in immobilized rat soleus muscles.

\section{Materials and Methods}

\section{Animals and experimental protocol (Fig. 1)}

Eighteen male Wistar rats (age, 8 weeks; weight, 311.0 $\pm 35.6 \mathrm{~g})$ were divided randomly into contol $(\mathrm{n}=3)$ and experimental $(\mathrm{n}=15)$ groups. Control rats were untreated. Animals from the experimental group were anesthetized with a pentobarbital sodium and bilateral ankles were fixed in full planter flexion by a plaster cast, the soleus muscles being immobilized in the shortened position. The plaster cast, worn from above of the knee joint to the distal foot, was changed weekly because of loosening that occurred secondary to muscle atrophy ${ }^{8}$. After the experimental group rats were immobilized for 4 weeks, animals were divided into three groups. One group which received immobilization alone were assigned to the immobilization group (group I, n=3). The other two groups were immobilized for 4 weeks, and then underwent two different remobilization program: one group was allowed to move freely in their cages and underwent stretch training for 1 or 3 weeks (group $S, n=6$ ), the other group was allowed to move freely in their cages but did not underwent stretch training for 1 or 3 weeks (group NS, n=6). Using nonelastic tape, both soleus muscles of the rats of group $\mathrm{S}$ were maximally stretched by dorsiflexing the ankle joint under anesthesia for $30 \mathrm{~min}$ a day ${ }^{7}, 6$ times a week. Rats of group NS were anesthetized for only $30 \mathrm{~min}$ a day, 6 times a week. At the end of the experimental periods, each rat was anesthetized with a pentobarbital sodium. The body weight was measured, and the soleus muscle was extracted from each hindlimb. The experimental protocol was approved by the Ethics Review Committee for Animal Experimentation at our institution ${ }^{9)}$.

\section{Histological and histochemical methods}

Extracted muscle samples were weighed and frozen in isopentane cooled to the freezing point with liquid nitrogen. Serial cross sections of $10 \mu \mathrm{m}$ were cut on a cryostat (type FS/FAS, Bright Ltd., Great Britain) then stained with hematoxylin-eosin and myofibrillar adenosine triphosphatase (ATPase, $\mathrm{pH}$ 10.8).

\section{Data analysis}

Range of ankle joint movement: Immediately after immobilization for 4 weeks and subsequent remobilization for 1,2 , or 3 weeks, the range of ankle joint movement on dorsiflexion was measured with a goniometer. To do this,

\begin{tabular}{|c|c|c|c|c|c|c|c|}
\hline Groups & $\mathrm{n}$ & $\begin{array}{l}\text { (Age) } \\
\text { (Week) }\end{array}$ & $\begin{array}{l}8 \mathrm{~W} \\
0 \mathrm{~W}\end{array}$ & $\begin{array}{l}12 \mathrm{~W} \\
4 \mathrm{~W}\end{array}$ & $\begin{array}{l}13 \mathrm{~W} \\
5 \mathrm{~W}\end{array}$ & $\begin{array}{l}14 W \\
6 \mathrm{~W}\end{array}$ & $\begin{array}{l}15 \mathrm{~W} \\
7 \mathrm{~W}\end{array}$ \\
\hline Control & 3 & & & & & & \\
\hline Group I & 3 & & & & $\mathrm{n}=3$ & & $n=3$ \\
\hline Group NS & 6 & & & & & & $y$ \\
\hline Group $\mathrm{S}$ & 6 & & & & & & \\
\hline
\end{tabular}

Fig. 1. Experimental protocol

the anesthesized rat was laid on its side and its knee joint flexed about 90 degrees. The ankle was passively dorsiflexed maximally and measurment made of the form angle (0-180 degrees) of the line knots of the 5th metatarsale and the malleolus lateralis of the fibula, and the line knots the malleolus lateralis of the fibula and the center of the knee joint.

Muscle fiber diameter and fiber type distribution: Analysis was performed with an Apple Power Macintosh personal computer (9500/132) and the public domain NIH Image program (Ver. 1.59). A minimum of 100 fibers in each muscle was used to determine the diameters of the fibers and percentage distribution of fiber types.

Statistical analysis: A non-paired Student's t-test and a one-way analysis of variance (ANOVA) were used for the statistical analysis. If significance was obtained $(p<0.05)$ by the ANOVA, pairwise comparisons were made by Scheffe's F method.

\section{Results}

\section{Range of ankle joint movement}

In groups I, NS, and S, the range of ankle joint movement on dorsiflexion immediately after immobilization was reduced significantly as compared with the controls, but there was no significant difference between the three groups. After 1 week of remobilization, the range of ankle joint movement on dorsiflexion in groups NS and S had increased significantly compared to that immediately after immobilization, but there was no significant difference between groups NS and S after 1, 2, or 3 weeks of remobilization (Fig. 2).

\section{Muscle wet weight}

Muscle wet weight for group I was decreased significantly compared with that for the controls. After 1 and 3 weeks of remobilization, the muscle wet weights for groups NS and S had increased significantly as compared with group I, but there was no significant difference between groups NS and S (Table 1).

Since the muscle wet weight is affected by body weight, our study therefore was determined changes in the relative weight of soleus muscles. After 1 week of 


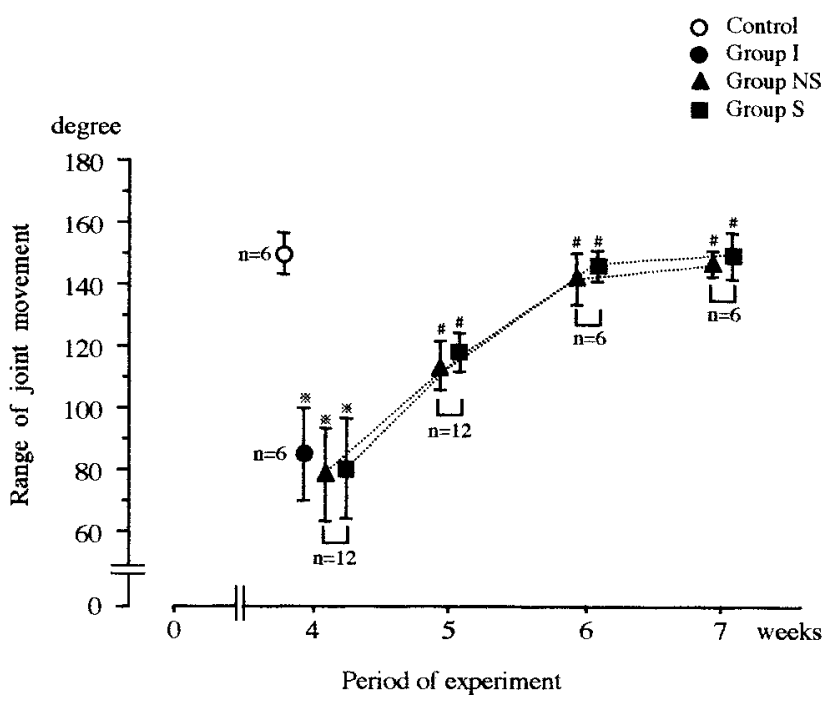

Fig. 2. Change in the range of ankle movement on dorsiflexion. Values are means \pm SD. $\mathrm{n}$; number of ankle joints, *; $\mathrm{p}<0.01$ (compared with control), \#; $\mathrm{p}<0.01$ (compared with values immediately after immobilization for each group).

remobilization, the weight of the soleus muscle relative to body weight for group $S$ had increased significantly as compared with group I, but group NS did not show a significant difference as compared with group I. After 3 weeks of remobilization, groups NS and S had increased significantly as compared with group I (Table 1).
Muscle fiber diameter

In group I, the means of the muscle fiber diameters for types I and II fibers decreased significantly as compared with those for the control, groups NS and S. After 1 week of remobilization, the means of the muscle fiber diameters for types I in group S had increased significantly compared with group NS, but those for type II fibers in group S did not significantly differ from that for group NS. After 3 weeks of remobilization, the means of the muscle fiber diameters for types I and II fibers in group $\mathrm{S}$ had increased significantly compared with group NS. (Fig. 3 and Table 2).

A high percentage of type II fiber but less type I fiber were present in groups I, NS, and S as compared with the control, but there was no significant difference between the experimental group (Table 2).

\section{Discussion}

In general, stretch training has been underwent for recovery and/or prevent from the muscle atrophy, the decrease of extensibility at muscle and soft tissue, and the decrease of the range of movement on physical therapy programs. However, it is doubtful whether the competent muscle is stretched enough when its range of movement is decreased by joint contracture. The major purpose of the present study has been to determine whether short duration stretching is ameliorating for disuse muscle atrophy that has been followed by joint contracture.

Table 1. Muscle wet weight and weight of the soleus muscle relative to body

\begin{tabular}{|c|c|c|c|c|c|c|}
\hline \multirow{2}{*}{$\begin{array}{c}\text { Groups } \\
\text { (Number of muscles) }\end{array}$} & \multirow[b]{2}{*}{ Control (6) } & \multirow[b]{2}{*}{ Group I (6) } & \multicolumn{2}{|c|}{ After 1 week of remobilization } & \multicolumn{2}{|c|}{ After 3 weeks of remobilization } \\
\hline & & & Group NS (6) & Group S (6) & Group NS (6) & Group S (6) \\
\hline Muscle wet wt (mg) & $225.3 \pm 35.6$ & $98.5 \pm 14.3^{*}$ & $167.2 \pm 33.0^{*, \#}$ & $164.0 \pm 30.3^{*, \#}$ & $167.5 \pm 6.0^{*, \#}$ & $170.7 \pm 10.9^{*, \#}$ \\
\hline \multicolumn{7}{|l|}{ Muscle wet wt (mg) } \\
\hline Body wt (g) & $0.54 \pm 0.08$ & $0.32 \pm 0.04 *$ & $0.44 \pm 0.09$ & $0.48 \pm 0.06^{\#}$ & $0.48 \pm 0.03^{\#}$ & $0.48 \pm 0.04^{\#}$ \\
\hline
\end{tabular}

Values are mean \pm SD.

*; $\mathrm{p}<0.05$ (compared with control), ; $\mathrm{p}<0.05$ (compared with group I).

Table 2. Muscle fiber diameter and fiber type distribution

\begin{tabular}{|c|c|c|c|c|c|c|}
\hline \multirow{2}{*}{$\begin{array}{c}\text { Groups } \\
\text { (Number of muscles) }\end{array}$} & \multirow[b]{2}{*}{ Control (6) } & \multirow[b]{2}{*}{ Group I (6) } & \multicolumn{2}{|c|}{ After 1 week of remobilization } & \multicolumn{2}{|c|}{ After 3 weeks of remobilization } \\
\hline & & & Group NS (6) & Group S (6) & Group NS (6) & Group S (6) \\
\hline \multicolumn{7}{|c|}{ Fiber diameter by type $(\mu \mathrm{m})$} \\
\hline Type I & $57.0 \pm 15.7$ & $36.6 \pm 11.0^{*}$ & $54.1 \pm 11.6^{\#}$ & $56.4 \pm 11.5^{\#, \uparrow}$ & $49.3 \pm 10.9^{\#}$ & $56.4 \pm 13.7^{\#, \dagger}$ \\
\hline Type II & $48.9 \pm 10.2$ & $29.0 \pm 8.2 *$ & $43.3 \pm 8.8^{\#}$ & $44.3 \pm 8.7^{\#}$ & $40.7 \pm 8.9^{\#}$ & $42.3 \pm 10.7^{\#, \dagger}$ \\
\hline \multicolumn{7}{|c|}{ Fiber type distribution (\%) } \\
\hline Type I & $87.8 \pm 2.2$ & $71.0 \pm 4.3^{*}$ & $70.8 \pm 3.2 *$ & $68.1 \pm 3.9^{*}$ & $74.1 \pm 4.7 *$ & $73.5 \pm 4.7 *$ \\
\hline Type II & $12.2 \pm 2.2$ & $29.0 \pm 4.3^{*}$ & $29.2 \pm 3.2 *$ & $31.9 \pm 3.9^{*}$ & $25.9 \pm 4.7 *$ & $26.5 \pm 4.7 *$ \\
\hline
\end{tabular}

Values are mean \pm SD.

*; $p<0.05$ (compared with control), ${ }^{\#} ; \mathrm{p}<0.05$ (compared with group I), ${ }^{\dagger} ; \mathrm{p}<0.05$ (compared with group NS). 

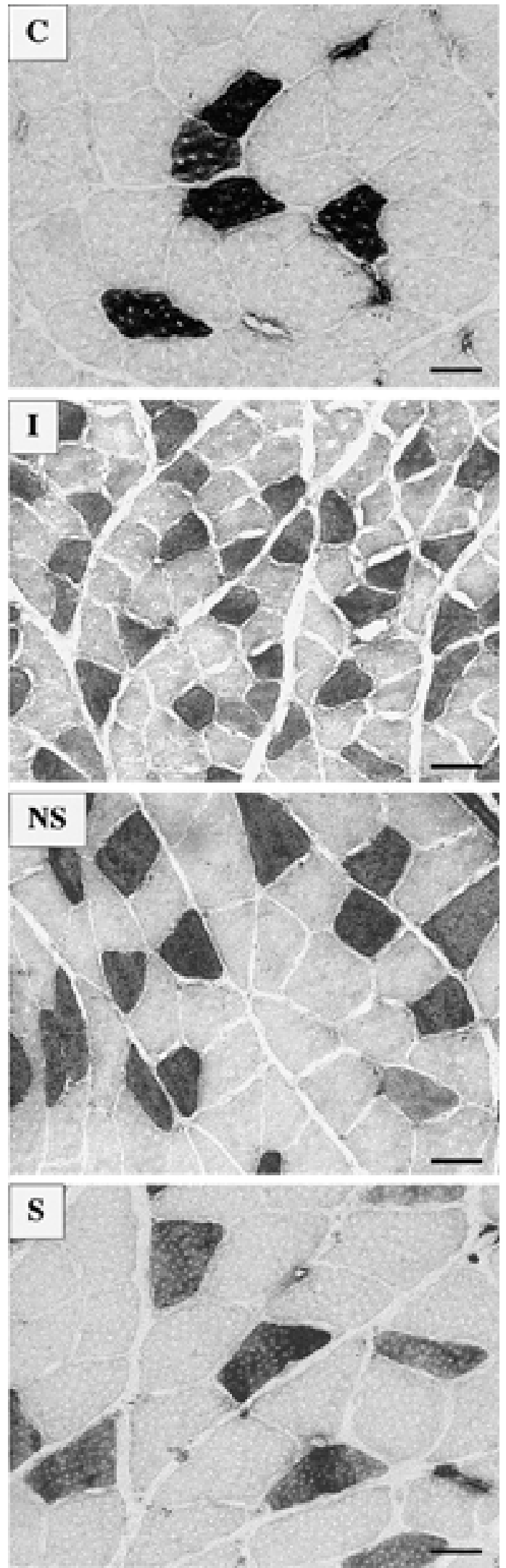

Fig. 3. Myofibrillar ATPase (pH 10.8) activity was used to discriminate type I (light stain) and II (dark stain) fibers. Bar $=50 \mu \mathrm{m}$.

C; Control, I; Group I, NS; Group NS, after 3 weeks of remobilization, S; Group S, after 3 weeks of remobilization.
In groups I, NS, and S, bilateral ankles were immobilized throughout 4 weeks in full planter flexion by a plaster cast. Immediately after immobilization, the range of ankle joint movement on dorsiflexion in these group was greatly reduced, but there was no significant difference between the three groups. This result indicated that ankle joint contracture was similar in all three groups. After 1 week of remobilization, the range of ankle joint movement on dorsiflexion in groups NS and S greatly increased compared to that immediately after immobilization, but there was no significant difference between them after 1,2, or 3 weeks of remobilization. Williams reported that daily period of intermittent stretch lasting $30 \mathrm{~min}$ or more were found to maintain normal dorsiflexion of the ankle which occurs in mouse soleus muscle immobilization ${ }^{7}$. In our study, daily period of short duration stretching was decided in the light of this report ${ }^{7}$, but there was no difference in recovery from ankle joint contracture between stretch training and spontaneous recovery. The reason for this results can not refer, but we are often experienced what the joint contracture improve by stretch training in clinical. We therefore need to consider that the effect of various period of short duration stretching in future.

Group I showed decreased muscle wet weight, the weight of the soleus muscle relative to body weight, the means of the muscle fiber diameters for types I and II fibers, and tended to have a high percentage of type II fiber and less type I fiber. These results suggested that rat soleus muscles immobilized throughout 4 weeks induced muscle fiber atrophy in both types I and II fibers.

After 1 week of remobilization, the muscle wet weight, and the means of the muscle fiber diameters for types I and II fibers in groups NS and S had increased significantly compared with group I. Also, the means of the muscle fiber diameters for type I in group S had increased significantly compared with group NS, but those for type II fibers in group S did not significantly differ from that for group NS. Furthermore, after 3 weeks of remobilization, the means of the muscle fiber diameters for types I and II fibers in group $\mathrm{S}$ had increased significantly compared with group NS. These results therefore indicated that when disuse atrophy in immobilized rat soleus muscles were subjected to short duration stretching, only type I fiber accelerated hypertrophy for a 1-week period and that both type I and II fibers accelerated hypertrophy for a 3-week period, rather than the spontaneous recovery.

Yamazaki et al. ${ }^{56)}$ and Williams ${ }^{7)}$ reported that short duration stretching prevents the progression of disuse muscle atrophy. Furthermore, increased protein synthesis occurs in stretched muscle measured in vivo or in vitro after appropriate plaster cast immobilization ${ }^{410)}$. Inui et al. reported that muscle stretching can be increased for twitch tension in slow twitch fibers of rat soleus muscles ${ }^{11)}$. Yang et al. reported that passive stretching by plaster cast 
immobilization of the muscle in its lengthened position not only induced an increase in insulin-like growth factor I (IGF-I) mRNA expression within individual muscle fibers but an increase in the percentage of fibers expressing neonatal and slow myosin, and that this change in phenotype was accompanied by a rapid, marked increase in muscle mass and total RNA content, as well as in IGF-I gene expression ${ }^{12)}$. They suggested that IGF-I expression in individual muscle fibers is correlated not only with hypertrophy but with the muscle phenotypic adaptation that results from stretch and overload ${ }^{12)}$. Their recent findings have clarified the mechanism of muscle fiber hypertrophy caused by muscle stretching. Our findings suggest that short duration stretching can induce recovery from disuse muscle atrophy that has been followed by joint contracture.

On the other hand, a high percentage of type II fiber but less type I fiber were present in groups NS and S after 1 and 3 weeks of remobilization. No difference in the fiber type distribution were observed between the experimental group. Yamazaki et al. ${ }^{5) 6)}$ reported that the change of fiber type distribution on disuse atrophy of rat soleus muscles induced by hindlimb suspension can not be prevented, by maintaining the stretch position for $20 \mathrm{~min}$ a day. Our results also indicated that short duration stretching can not induce recovery from the change of fiber type distribution in immobilized soleus muscles. We therefore need to consider that the critical amount of stretch, duration required to produce maximal effects, and the various methods of stretch in future.

In conclusion, when disuse atrophy in immobilized rat soleus muscles were subjected to short duration stretching for 30 min a day, muscle fiber hypertrophy can be accelerated, rather than the spontaneous recovery. We therefore presume that stretch training may be effective therapy for recovery from disuse muscle atrophy that has been followed by joint contracture.

\section{References}

1) Ikai $T$, Yonemoto $K$, et al.: Basic research on muscle and bone atrophies - Relation with clinical medicine-. Rigaku ryohogaku 23: 444-447, 1996 (in Japanese).

2) Yonemoto K: Disuse (immobilized) muscle atrophy. In: Hizawa K, Nonaka I, et al. (eds) Muscular Pathology 2nd ed, Tokyo, Bunkodo, 1989, pp 381-388 (in Japanese).

3) Baker JH, Matsumoto DE: Adaptation of skeletal muscle to immobilization in a shortened position. Muscle Nerve 2: 213-244, 1988.

4) Goldspink G, Williams PE: Muscle fibre and connective tissue changes associated with use and disuse. In: Ada L, Canning C (eds) Key Issues in Neurological Physiotherapy. 8th ed, Oxford, Butterworth-Heinemann, 1990, pp 197-218.

5) Yamazaki $\mathrm{T}$, Tachino $\mathrm{K}$, et al:: Effect of short duration stretching under anesthesia in preventing disuse muscle atrophy in rats. Rigaku ryōhōjanaru 29: 135-138, 1995 (in Japanese).

6) Yamazaki $\mathrm{T}$, Tachino $\mathrm{K}$, et al: : Effect of short duration stretching for prevention of disuse muscle atrophy in mature rats. Rigaku ryōhōgaku 23: 349-355, 1996.

7) Williams PE: Use of intermittent stretch in the prevention of serial sarcomere loss in immobilised muscle. Ann Rheu Dis 49: 316-317, 1990.

8) Okita M, Yoshimura T, et al.: The effect of immobilization on collagen fibrillar arrangements in the endomysium of the rat soleus muscle. Rigaku ryōhōgaku 24: 23-30, 1997 (in Japanese).

9) Guidelines for Animal Experimentation Nagasaki University. In: Laboratory Animal Center for Biomedical Research, Nagasaki University School of Medicine, User's Guidebook, 1995, pp 24-33 (in Japanese).

10) Goldspink DF: The influence of immobilization and stretch on protein turnover of rat skeletal muscle. J Physiol 264: 267282, 1977.

11) Inui $K$, Takeda $H$, et al.: Effects of the stretching on the rat soleus muscle. Rigaku ryōhōgaku 23: 452-455, 1996 (in Japanese).

12) Yang $H$, Aluaqeeb $M$, et al.: Change in muscle fibre type, muscle mass and IGF-I gene expression in rabbit skeletal muscle subjected to stretch. J Anat 190: 613-622, 1997. 